\title{
BIOLOGICAL PEST CONTROL BY USING A COMPETITIVE SPECIES AND WITH PROVISION OF ADDITIONAL FOOD
}

\author{
M.S. Bhuvaneswari ${ }^{1}$, B.S.R.V. Prasad ${ }^{2} \S$ \\ ${ }^{1,2}$ Department of Mathematics \\ School of Advanced Sciences \\ VIT University \\ Vellore, TN 632014, INDIA
}

\begin{abstract}
Biological pest control is the eco-friendly method of controlling pests using natural enemies such as predators, pathogens, and parasitoids. It is one of the important components of IPM. Recent works on biological control focusing on the aspects of controlling economically damaging pest by introducing non-economically damaging pests as competitors. One of the ways to enhance the biological control is to provide suitable additional/alternative food to competitors. In this article, we study the dynamics of controlling the economically damaging pest species by providing additional food to non-economically damaging competitive pests. Mathematically these competitive interactions are modelled using Lokta-Volterra model of competition and with Holling type II functional response towards the additional food. The conditions on the quality and quantity of additional food under which successful biological control can be achieved have been obtained. The global dynamics of the systems have studied by analyzing various bifurcations that are occurring in the system. The study can aid the eco-managers, on the choice of competitive species and the additional food for accomplishing successful biological control. The results of the theoretical observations are in agreement with few field experiments.
\end{abstract}

AMS Subject Classification: 92D25, 93A30, 93B05, 93B52, 49J30

Key Words: pest, inter-specific competition, biological control, additional food

Received: May 24, 2016

Published: September 10, 2016

${ }^{\S}$ Correspondence author (c) 2016 Academic Publications, Ltd. url: www.acadpubl.eu 


\section{Introduction}

Controlling the economically damaging agricultural pest species, in particularly, weeds and insects pests, has been attracting lot of attention from the ecomanagers long since. Managing the agricultural pest became a vital task to coup up with increasing human population [18]. Traditional approaches to control the pest such as spraying insecticides, pesticides is found to have long-lasting side affects as they contaminate the environment. It also found that insects such as diamondback moth, Plutella xylostealla, have developed resistance to all available chemicals $[7,18]$. Due to this hazardous affects of the chemical control, the eco-managers are moving towards other alternative control strategies such as bio-remediation and bio-control to safeguard the environment [14].

It has been recognized long back that most of the natural enemies are omnivores and thus feed on pest as well as other plant-provided food such as nectar, pollen [23]. Lot of experimental works carried out in this direction and important conclusions have been derived on the use of non-prey food sources (viz,. nectar, pollen) to enhance the biological control efficiency of the natural enemies in the agricultural fields $[11,12,15,33]$. The conditions on the quality and quantity of the additional food that is to be provided to be predators for successful biological control have been analyzed in [28, 29, 30, 31].

The another important aspect, which is common in the biological system is the competition between the species, which can be either direct or indirect interaction between two consumers for food, resources, space etc. [13, 22, 20]. The competition can mainly be classified into (i) intra-specific competition, the competition that is occurring between same taxa and (ii) inter-specific competition, the type of competition that is occurring between different animal/plant groups. It is found that in many biological system intra-specific competition is evident and predominant $[3,6,19,20,24,25,32,35]$. Recently, many ecologists are exploiting this phenomenon and experimenting with controlling the economically damaging pest species by introducing another non-economically damaging pest species, which is a competitor for the former species [19, and refernces there in]. It is pointed out by the authors that although the early attempts to control the Pacific spider mites by introducing Willamette mites has been successful, the mechanisms that are involved in this are poorly understood. This motivated us to develop a mathematical model of competing pest species in which non-economically damaging pest species are provided with some additional food of constant biomass and examine the conditions under which the economically damaging pest species can be eradicated/brought down to the levels at which they are not damaging the crop. By characterizing the 
additional food with respect to its quality and quantity, conditions have been derived that will lead to eradication of economically damaging pest.

In this article, we consider a modified version of the classical Lokta-Volterra competitive system [20] to examine the interactions of competing species in presence of additional food to one of the species. We assume that the additional food is not reproductive in nature, but is maintained at a constant biomass either by nature or external agency. This assumption reduces the dimension of the considered system, by which it is possible to study the role of additional food using phase plane analysis $[29,28]$. We further assume that, the number of encounters per species (to which additional food is provided) with the additional food is proportional to the density of the additional food and will not be affected by the other competing species. By analyzing the dynamics of the proposed model in a systematic way, the vital parameters that influence the dynamics have be identified and controllability of the system is discussed. This study provides the management strategies that are to be followed for the success of biological pest control in a competitive system in which one of the species is provided with additional food.

The section division of article is as follows: Section 2 reviews the classical Lotka-Volterra model of interspecific competitive model. In Section 3, the model representing the dynamics of competitive system in presence of additional food is analyzed and equilibrium analysis is presented. The global dynamics of the considered model are presented in Section 4. The consequences of providing additional food competitive system and its implications to biological control has been presented in Section 5 and followed by discussion and conclusions in Section 6 .

\section{The Lokta-Volterra Model of Interspecific Competition Model}

Let us consider the following Lokta-Volterra model of interspecific competition between two species $N_{1}$ and $N_{2}$. Let us assume that $N_{1}$ denotes the population density of economically damaging pest and $N_{2}$ denotes the population density of non-economically damaging pest.

$$
\begin{aligned}
& \frac{d N_{1}}{d T}=r N_{1}\left(1-\frac{N_{1}}{k}\right)-b N_{1} N_{2}, \\
& \frac{d N_{2}}{d T}=s N_{2}\left(1-\frac{N_{2}}{l}\right)-c N_{1} N_{2} .
\end{aligned}
$$

Here $r$ denotes the intrinsic growth rate of economically damaging pest $N_{1}$ and $s$ denote the intrinsic growth rate of non-economically damaging pest $N_{2}$. We 
assume here that, the both species $N_{1}$ and $N_{2}$ grows Logistically in the absence of other with their respective carrying capacities being $k, l$ respectively. $b(c)$ denotes the interspecific competition affect of $N_{2}\left(N_{1}\right)$ on $N_{1}\left(N_{2}\right)$. The nondimensionalized version of the system $(1,2)$ is given by:

$$
\begin{aligned}
& \frac{d x}{d t}=x(1-x)-\beta_{1} x y, \\
& \frac{d y}{d t}=R y(1-y)-\beta_{2} x y .
\end{aligned}
$$

The model $(3,4)$ is well studied in the literature and analyzed from various perspectives [20]. The model $(3,4)$ always admit the trivial equilibrium $E_{0}=$ $(0,0)$ and two axial equilibria $E_{1}=(1,0), E_{2}=(0,1)$. The existence of interior equilibrium $E^{*}=\left(x^{*}, y^{*}\right)$ where

$$
x^{*}=\frac{R\left(1-\beta_{1}\right)}{R-\beta_{1} \beta_{2}}, \quad \text { and } \quad y^{*}=\frac{R-\beta_{2}}{R-\beta_{1} \beta_{2}}
$$

for the system is conditional dependent. If the parameters of the system satisfies any of the following conditions,

Case 1: $\frac{1}{\beta_{1}}<1, \frac{R}{\beta_{2}}<1$

Case 2: $\frac{1}{\beta_{1}}>1, \frac{R}{\beta_{2}}>1$

then the considered system admits unique interior equilibrium. The considered system does not admits any interior equilibrium if the parameters of the system satisfies any of the following condition

Case 3: $1>\frac{1}{\beta_{1}}, 1<\frac{R}{\beta_{2}}$

Case 4: $1<\frac{1}{\beta_{1}}, 1>\frac{R}{\beta_{2}}$

The following theorems illustrate the nature of the existing equilibria, which will be useful in our further analysis.

Lemma 1. The trivial equilibrium point $(0,0)$ is always an unstable node.

Lemma 2. The axial equilibrium point $(1,0)$ is stable node (saddle) in nature if $R-\beta_{2}<(>) 0$.

Lemma 3. The axial equilibrium point $(0,1)$ is stable node (saddle) in nature if $1-\beta_{1}<(>) 0$.

Lemma 4. The interior equilibrium $\left(x^{*}, y^{*}\right)$ is stable if $R-\beta_{1} \beta_{2}>0$ and is saddle if $R-\beta_{1} \beta_{2}<0$. 


\section{Model in Presence of Additional Food to Non-Economically Damaging Pest}

Let us now assume that, the non-economically damaging species $N_{2}$ is provided with an additional food of constant biomass $A$, which is distributed uniformly in the environment. We also assume that the number of encounters of noneconomically damaging species $N_{2}$ with the additional food is proportional to the density of the additional food and $N_{2}$ species are not optimal foragers. We further assume that the interaction of $N_{2}$ with additional food does not affect the competitive interaction rates of $N_{1}$ over $N_{2}$ and $N_{2}$ over $N_{1}$. With this assumptions and using the time utilization concept, the functional response of non-economically damaging species towards the available additional food is given by

$$
g\left(N_{2}, A\right)=\frac{A}{\eta_{1}+h_{A} A}
$$

where $\eta_{1}=\frac{1}{e_{A}}$. Incorporating the above expression for the functional response of non-economically damaging pest $N_{2}$ towards additional food, we obtain the following coupled differential system representing dynamics of two competing species $N_{1}, N_{2}$, when the $N_{2}$-species is provided with additional food.

$$
\begin{aligned}
& \frac{d N_{1}}{d T}=r N_{1}\left(1-\frac{N_{1}}{k}\right)-b N_{1} N_{2} \\
& \frac{d N_{2}}{d T}=s N_{2}\left(1-\frac{N_{2}}{l}\right)-c N_{1} N_{2}+\frac{A N_{2}}{\eta_{1}+h_{A} A}
\end{aligned}
$$

Here $r, s$ denote the intrinsic growth rate of $N_{1}, N_{2}$ species respectively. $k, l$ are the carrying capacities of $N_{1}, N_{2}$ species. $b(c)$ competitive interaction rate $N_{2}\left(N_{1}\right)$ over $N_{1}\left(N_{2}\right)$. A denotes the quantity of additional food that is supplied to $N_{2}$ species. $h_{A}$ is the handling time of $N_{2}$ species towards the additional food.

It is easy to observe that the above system reduces to the Lokta-Volterra system of competing species $(1,2)$, when $A$ is taken to be zero. We now analyze the dynamics of the system $(7,8)$.

Before proceeding to the further analysis, we reduce the number of parameters in the considered system by the process of non-dimensionalisation. Introducing the transformation,

$$
x=\frac{N_{1}}{k}, y=\frac{N_{2}}{l}, t=r T,
$$

the system $(7,8)$ gets reduce to

$$
\frac{d x}{d t}=x(1-x)-\beta_{1} x y
$$




$$
\frac{d y}{d t}=R y(1-y)-\beta_{2} x y+\frac{\xi y}{1+\alpha \xi}
$$

The $x$-species zero-growth isoclines for the system are given by

$$
x=0, \quad \text { and } \quad y=\frac{1}{\beta_{1}}(1-x) .
$$

The $y$-species zero-growth isoclines for the system are given by

$$
y=0, \quad \text { and } \quad y=\frac{-\beta_{2} x}{R}+\left(1+\frac{\xi}{R(1+\alpha \xi)}\right) .
$$

It can be easily observed that the non-trivial $x$-nullcline is a straight line, with a negative slope of $\frac{1}{\beta_{1}}$, which intersects the $x$-axis at $(1,0)$ and $y$-axis at $\left(0, \frac{1}{\beta_{1}}\right)$. The non-trivial $y$-nullcline is also a straight line with a slope of $-\frac{\beta_{2}}{R}$, and which is a function of $\alpha$ and $\xi$. This line intersects $x$-axis at $(\bar{x}, 0)$ and $y$-axis at $(0, \bar{y})$ where,

$$
\bar{x}=\frac{1}{\beta_{2}}\left(R+\frac{\xi}{1+\alpha \xi}\right)
$$

and,

$$
\bar{y}=\left(1+\frac{\xi}{R(1+\alpha \xi)}\right) .
$$

From the properties of nullclines of the system, it can be easily verified that the system always admits the trivial equilibrium $E_{0}=(0,0)$ and two axial equilibrium points $E_{1}=(1,0), \quad E_{2}=(0, \bar{y})$.

By equating the non-trivial nullclines of the system, we obtain the interior equilibrium $E^{*}=\left(x^{*}, y^{*}\right)$, where

$$
\begin{aligned}
& x^{*}=\frac{R(1+\alpha \xi)\left(1-\beta_{1}\right)-\beta_{1} \xi}{(1+\alpha \xi)\left(R-\beta_{1} \beta_{2}\right)}, \\
& y^{*}=\frac{(1+\alpha \xi)\left(R-\beta_{2}\right)+\xi}{(1+\alpha \xi)\left(R-\beta_{1} \beta_{2}\right)} .
\end{aligned}
$$

From the equations $(15),(16)$, we can infer that the existence of $E^{*}=\left(x^{*}, y^{*}\right)$ is conditional dependent and accordingly, we have the following conditions:

1. The system admits no interior equilibrium if the parameters of the system satisfies:
(i) $R-\beta_{1} \beta_{2}>0$ and $\bar{x}<1, \bar{y}<\frac{1}{\beta_{1}}$
$[\mathrm{or}]$
(ii) $R-\beta_{1} \beta_{2}<0$ and $\bar{x}>1$, $\bar{y}>\frac{1}{\beta_{1}}$ 
2. The system admits unique interior equilibrium if the parameters of the system satisfies:

(i) $R-\beta_{1} \beta_{2}>0$ and $\bar{x}>1, \bar{y}<\frac{1}{\beta_{1}} \quad$ [or] $\quad$ (ii) $R-\beta_{1} \beta_{2}>0$ and $\bar{x}<1$,
$\bar{y}>\frac{1}{\beta_{1}}$

The associated community matrix $J_{(x, y)}$ for the considered system is given by,

$$
J_{(x, y)}=\left(\begin{array}{cc}
1-2 x-\beta_{1} y & -\beta_{1} x \\
-\beta_{2} y & R-2 R y-\beta_{2} x+\frac{\xi}{(1+\alpha \xi)}
\end{array}\right) .
$$

Evaluating the community matrix at each of the boundary equilibria and analyzing the eigenvalues of the resulting characteristic equation, we obtain the following results related to the stability of the trivial and axial equilibria.

Lemma 5. The trivial equilibrium $E_{0}=(0,0)$ is always unstable node.

Lemma 6. The system undergoes transcritical bifurcation around $E_{1}$ if the parameters of the system satisfies $\left(R-\beta_{2}\right)(1+\alpha \xi)+\xi=0$.

Lemma 7. The system undergoes transcritical bifurcation around $E_{2}$ if the parameters of the system satisfies $R(1+\alpha \xi)\left(1-\beta_{1}\right)+\xi=0$.

\section{Global Dynamics}

In this section, we analyze the global dynamics of the system and draw conclusions regarding the controllability of the system treating $\alpha$ and $\xi$ as control parameters and treating $R, \beta_{1}, \beta_{2}$ as ecosystem parameters.

The, global dynamics of the additional food provided competitive system can be better understood using the following four cases illustrating the existence/non-existence of interior equilibrium of Lokta-Volterra model and its stability

Case (ia) $R-\beta_{1} \beta_{2}>0$ and $\beta_{2}>R$,

Case (ib) $R-\beta_{1} \beta_{2}>0$ and $\beta_{2}<R$,

Case (iia) $R-\beta_{1} \beta_{2}<0$ and $\beta_{1}<1$,

Case (iib) $R-\beta_{1} \beta_{2}<0$ and $\beta_{1}>1$,

along with the transcritical bifurcation curves at $(1,0)$ and $(0, \bar{y})$, given by

$$
\left(R-\beta_{2}\right)(1+\alpha \xi)+\xi=0
$$



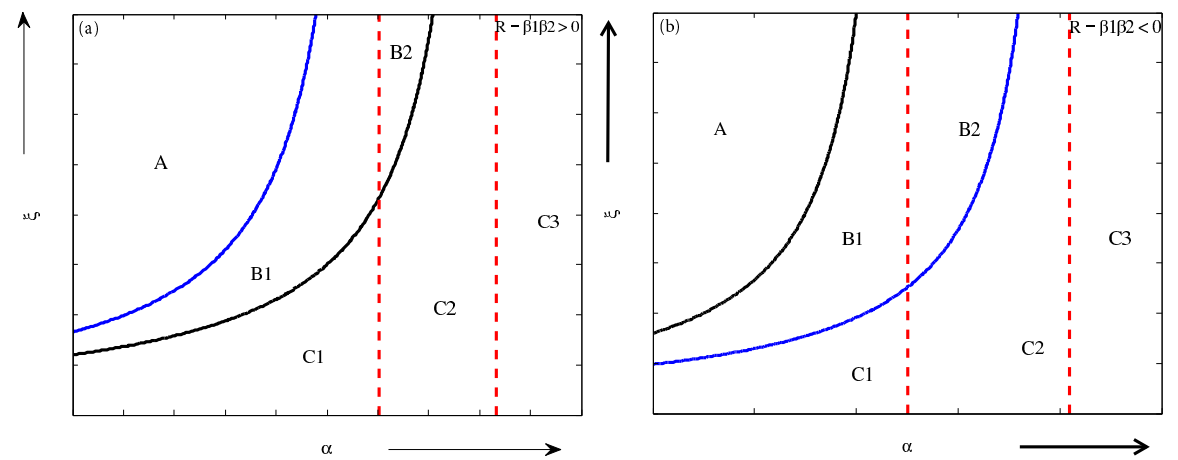

Figure 1: Bifurcation diagram in the $(\alpha, \xi)$ space for the case when the ecosystem parameters satisfy $R-\beta_{1} \beta_{2}>0$.

$$
R(1+\alpha \xi)\left(1-\beta_{1}\right)+\xi=0
$$

in positive $(\alpha, \xi)$ space. Note that in the case (ib), the curve (18) does not exist in the positive $(\alpha, \xi)$ space and in case (iib), the curve (19) does not exist in the positive $(\alpha, \xi)$. Thus, in these two cases the system dynamics entirely depends on the transcritical bifurcation that are occurring at $(0, \bar{y})$ and $(1,0)$ respectively. Observe that each of these curves (18), (19) when exist in the positive quadrant of $(\alpha, \xi)$ space, divide the positive quadrant into two regions, characterizing the nature of the associated equilibrium. It can be further observed that, $\alpha=\frac{1}{\beta_{2}-R}$ and $\alpha=\frac{\beta_{1}}{R\left(1-\beta_{1}\right)}$ are asymptotes for the curves (18), (19) respectively. The dynamics of the system under consideration, is studied through the curves (18) and (19), presented in the frames (a) and (b) of Fig. 1.

In Fig. 1a, the parameter space divided into three regions which are given by (i) $0<\alpha<\frac{\beta_{1}}{R\left(1-\beta_{1}\right)}$, (ii) $\frac{\beta_{1}}{R\left(1-\beta_{1}\right)}<\alpha<\frac{1}{\beta_{2}-R}$ and (iii) $\alpha>\frac{1}{\beta_{2}-R}$. Observe that cases (ii) and (iii) are only possible for $\beta_{2}>R$. When $\beta_{2}<R$, we have $\alpha>\frac{\beta_{1}}{R\left(1-\beta_{1}\right)}$. In case (i) for a fixed $\alpha$, as we increase $\xi$ from zero, we enter into either region $C_{1}$ or $B_{1}$ depending on whether $\beta_{2}>R$ or $\beta_{2}<R$. In this region $C_{1}$, the considered system does not admit any interior equilibrium. Here, the system admit the axial equilibrium $(1,0)$ which is stable in nature. The nature of coexisting axial equilibrium $(0, \bar{y})$ is saddle and all the solutions will be driven to $(1,0)$. Therefore, the system dominated by the economically damaging pest. As we increase $\xi$ which satisfies $\xi>\frac{\beta_{2}-R}{1-\alpha\left(\beta_{2}-R\right)}$, we move from the region $C_{1}$ to region $B_{1}$, we have emergence of a stable interior equilibrium $\left(x^{*}, y^{*}\right)$ due to a transcritical bifurcation taking place at $(1,0)$. The coexisting axial equilibria $(1,0)$ and $(0, \bar{y})$ are saddle in nature. Moving from region $B_{1}$ to 
$A$, with further increase in $\xi$ satisfying $\xi>\frac{R\left(1-\beta_{1}\right)}{\beta_{1}-R \alpha\left(1-\beta_{1}\right)}$, there is an exchange of stability between $(0, \bar{y})$ and $\left(x^{*}, y^{*}\right)$ due to the transcritical bifurcation taking place at $(0, \bar{y})$. The nature of axial equilibrium $(1,0)$ remains saddle in this region and that all the solutions of the system will converge to $(0, \bar{y})$.

In case (ii) with $\beta_{2}>R$, as $\xi$ increases from zero, we enter into region $C_{2}$. The dynamics of this region is similar to region $C_{1}$. Further increase in $\xi>\frac{\beta_{2}-R}{1-\alpha\left(\beta_{2}-R\right)}$, we move from the region $C_{2}$ to $B_{2}$, with a transcritical bifurcation occurring at $(1,0)$, with exchange of stability between $(1,0)$ and interior equilibrium $\left(x^{*}, y^{*}\right)$. The stability of $(0, \bar{y})$ remains saddle in this region.

In case (iii) with $\beta_{2}>R$, as $\xi$ increases from zero, we enter into region $C_{3}$, and stays there for any $\xi>0$. The dynamics of the system in this region is similar to the dynamics of regions $C_{1}$ and $C_{2}$. So, all the solutions will be driven the state $(1,0)$ asymptotically.

In Fig. 1b, we divide the parameter space into three cases (i) $0<\alpha<\frac{1}{\beta_{2}-R}$, (ii) $\frac{1}{\beta_{2}-R}<\alpha<\frac{\beta_{1}}{R\left(1-\beta_{1}\right)}$ and (iii) $\alpha>\frac{\beta_{1}}{R\left(1-\beta_{1}\right)}$. Observe that the cases (ii) and (iii) are valid only for $\beta_{1}<1$. For $\beta_{1}>1$ we have these two cases merged into one single case $\alpha>\frac{1}{\beta_{2}-R}$. In case(i), we observe that for a fixed $\alpha$, increasing $\xi$ from zero, we enter into the region $C_{1}$ or $B_{1}$ depending on whether $\beta_{1}<1$ or $\beta_{1}>1$. In this region $C_{1}$, the system admit axial equilibrium $(1,0)$, which is stable in nature and $(0, \bar{y})$, which is saddle in nature. In this region the system does not admit any interior equilibrium. All the solutions will be driven to $(1,0)$. Moving from the region $C_{1}$ to $B_{1}$ by increasing $\xi$ with $\xi>\frac{R\left(1-\beta_{1}\right)}{\beta_{1}-R \alpha\left(1-\beta_{1}\right)}$, the interior equilibrium $\left(x^{*}, y^{*}\right)$, which is saddle in nature, comes into existence with a transcritical bifurcation occurring at $(0, \bar{y})$. The nature coexisting axial equilibrium $(1,0)$ is remains unchanged. The unstable manifold of $\left(x^{*}, y^{*}\right)$ divide the phase space into two regions, such that the solutions starting in the one region will be converges to $(1,0)$ and the solutions starting in the other region will be converges to $(0, \bar{y})$. Moving from the region $B_{1}$ to $A$, with increase $\xi>\frac{\beta_{2}-R}{1-\alpha\left(\beta_{2}-R\right)}$, a transcritical bifurcation at $(1,0)$ occurs, due to which there will be an exchange of stability between $(1,0)$ and $\left(x^{*}, y^{*}\right)$. The stability of $(0, \bar{y})$ remains stable in this region. Therefore, all the solutions will be converges to $(0, \bar{y})$.

In case (ii) with $\beta_{1}<1$, as $\xi$ increases from zero, we enter into region $C_{2}$, with similar dynamics as that of region $C_{1}$. Moving from the region $C_{2}$ to $B_{2}$ through increasing $\xi$, we have emergence of a saddle interior equilibrium $\left(x^{*}, y^{*}\right)$. The coexisting axial equilibria $(1,0)$ and $(0, \bar{y})$ are both stable in nature.

In case (iii) with $\beta_{1}<1$, as $\xi$ increases from zero, we enter into region $C_{3}$ 
and stays in the region forever. The dynamics of the system in this region is similar to the dynamics of regions $C_{1}$ and $C_{2}$ i.e., all the solutions will be driven the state $(1,0)$ asymptotically.

\section{Consequences of Providing Additional Food the Competitive System}

In this section, we study the affect of providing the additional food to noneconomically damaging species and its consequences on system dynamics. As observed in the global dynamics of the system takes two opposite directions depending on quality, $\alpha$ and quantity, $\xi$ of the additional food that is provided.

In the case, when the system with out additional food supports the stable coexistence of both the competing species (i.e., $R-\beta_{1} \beta_{2}>0$ and $\beta_{2}<R$ ), continuous supply of additional food with quality, $\alpha<\frac{\beta_{1}}{R\left(1-\beta_{1}\right)}$ and quantity, $\xi \in\left(0, \frac{R\left(1-\beta_{1}\right)}{\beta_{1}-R \alpha\left(1-\beta_{1}\right)}\right)$ decreases the equilibrium component of the economically damaging species from $\frac{1-\beta_{1}}{R-\beta_{1} \beta_{2}}$. Increasing the additional food supply beyond this interval i.e., $\xi>\frac{R\left(1-\beta_{1}\right)}{\beta_{1}-R \alpha\left(1-\beta_{1}\right)}$, leads to eradication of the economically damaging species from the system in a finite time. On the other hand, providing the additional food with quality, $\alpha>\frac{\beta_{1}}{R\left(1-\beta_{1}\right)}$ and quantity $\xi>0$, the stable coexistence of both species continues, with equilibrium component of economically damaging species decreasing from $\frac{R\left(1-\beta_{1}\right)}{R-\beta_{1} \beta_{2}}$ and that of non-economically damaging species increasing from $\frac{R-\beta_{2}}{R-\beta_{1} \beta_{2}}$.

In the case, when the system with out additional food admits interior equilibrium with saddle in nature (i.e., $R-\beta_{1} \beta_{2}<0$ and $\beta_{1}>1$ ), continuous supply of additional food with quality, $\alpha<\frac{1}{\beta_{2}-R}$ and quantity, $\xi \in\left(0, \frac{\beta_{2}-R}{1-\alpha\left(\beta_{2}-R\right)}\right)$, increases the equilibrium component of the economically damaging species from $\frac{R\left(\beta_{1}-1\right)}{\beta_{1} \beta_{2}-R}$ and thus expanding the region of attraction of $(0, \bar{y})$. Increasing the additional food supply beyond this interval i.e., $\xi>\frac{\beta_{2}-R}{1-\alpha\left(\beta_{2}-R\right)}$, leads to exchange of stability between the interior equilibrium and axial equilibrium $(1,0)$. This exchange of stability leads to eradication of the economically damaging species from the system in a finite time. On the other hand, providing the additional food with quality, $\alpha>\frac{1}{\beta_{2}-R}$ and quantity $\xi>0$, the saddle coexistence of both species continues, with equilibrium component of economically damaging species increasing from $\frac{R\left(\beta_{1}-1\right)}{\beta_{1} \beta_{2}-R}$ and that of non-economically damaging species decreasing from $\frac{\beta_{2}-R}{\beta_{1} \beta_{2}-R}$ and leading to increase in the region of attraction of $(0, \bar{y})$. 

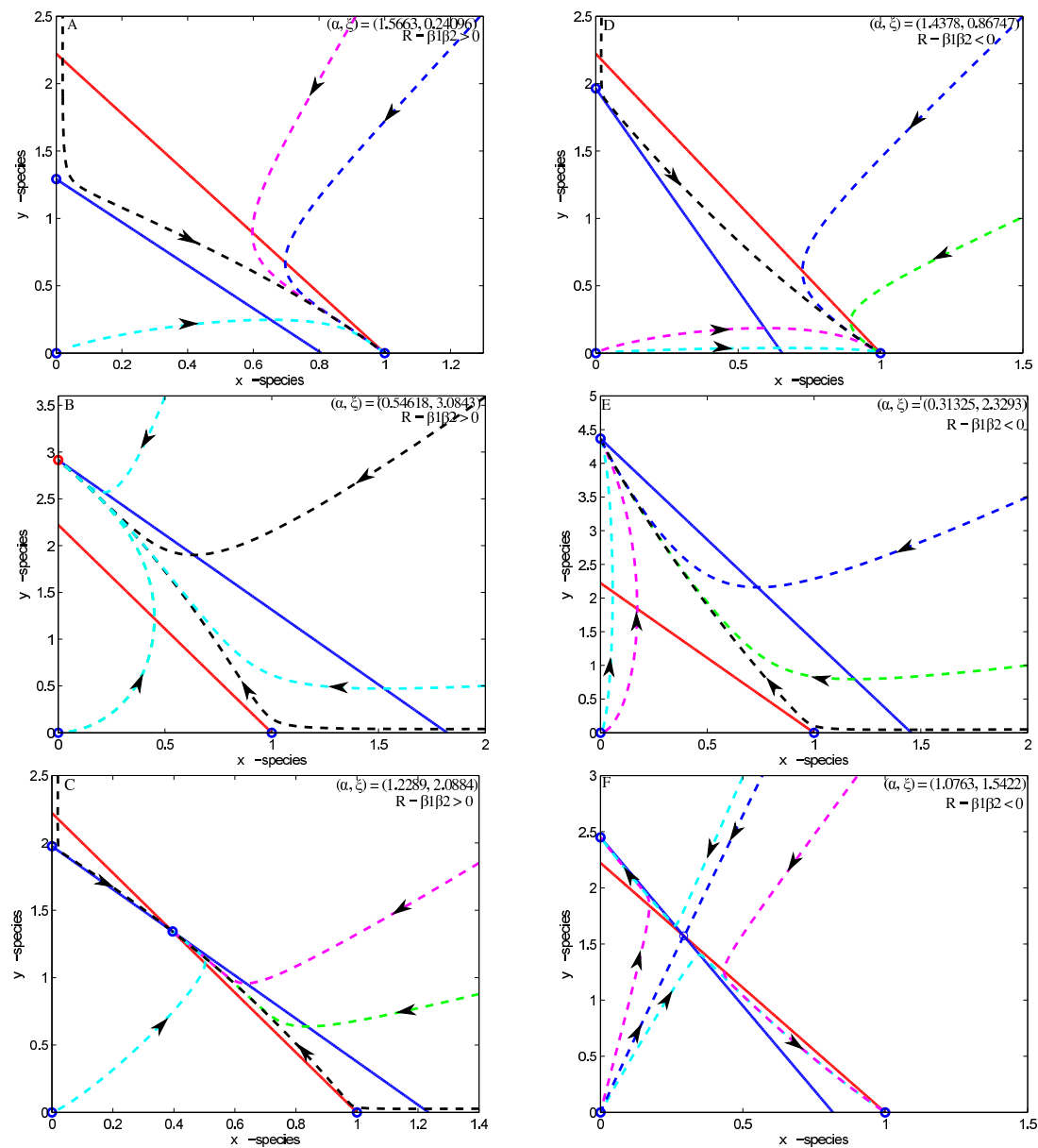

Figure 2: Numerical simulations illustrating the various dynamics and controllability aspects of the considered model. Frames A,D presents the case where the solutions will be driven the axial equilibrium $(1,0)$ and thus making the system pest dominated. The situation in which the solutions will be driven to a state, which is free from the economically damaging pest species is presented in frames B,E. Frames C,F presents the case where in the system admits an interior equilibrium. The equilibrium obtained in the case $R-\beta_{1} \beta_{2}>0(<0)$ is always stable (saddle) as noted in the consequences. 
In the case, when the system with out additional food does not support the coexistence and dominated by economically damaging species, the provision of additional food of quality $\alpha \in\left(0, \max \left\{\frac{1}{\beta_{2}-R}, \frac{\beta_{1}}{R\left(1-\beta_{1}\right)}\right\}\right)$ and quantity $\xi>\min \left\{\frac{R\left(1-\beta_{1}\right)}{\beta_{1}-R \alpha\left(1-\beta_{1}\right)}, \frac{\beta_{2}-R}{1-\alpha\left(\beta_{2}-R\right)}\right\}$ brings the coexistence of the two competing species. This coexistence will be stable if $R-\beta_{1} \beta_{2}>0$ and saddle if $R-\beta_{1} \beta_{2}<$ 0 . This coexistence ceases to exist if the quality $\alpha<\min \left\{\frac{1}{\beta_{2}-R}, \frac{\beta_{1}}{R\left(1-\beta_{1}\right)}\right\}$ and $\xi>\max \left\{\frac{R\left(1-\beta_{1}\right)}{\beta_{1}-R \alpha\left(1-\beta_{1}\right)}, \frac{\beta_{2}-R}{1-\alpha\left(\beta_{2}-R\right)}\right\}$. In this case all the solutions of the system will be driven to the state $(0, \bar{y})$, making system free from economically damaging species.

The key findings of the above discussions are illustrated through the numerical simulations presented in Fig. 2. The frames A,B,C of the Fig. 2 presents the case where $R-\beta_{1} \beta_{2}>0$ and frames D,E,F of the the Fig. 2 presents the case where $R-\beta_{1} \beta_{2}<0$.

\section{Discussion and Conclusions}

In this article, we have carried out a detailed analysis of dynamics of two competitive species, in presence of additional food to one of the species, with the assumption that additional food is provided to non-economically damaging pest species and the uptake of additional food is not affected the presence of economically damaging species. The additional food that is assumed to be of nonreproducing and it is distributed uniformly in the environment. The uptake of additional food by non-economically is modeled using Holling type II functional response. From the analysis of the model, it is observed that the quality, characterized by the handling times of non-economically damaging species towards the additional food and quantity of additional food plays a vital role in controlling system.

When the original system admits coexistence of two competing species (either a stable or saddle), providing additional food having specified characteristics, one can eliminate the economically damaging species from the system, achieving the needed biological control. In the case when the original system has non-coexistence of competing species, which is dominated by economically damaging species, providing additional food has several subcases are possible. In this case, the coexistence can be brought into the system by providing the non-economically damaging species with additional food, whose quality and quantity levels belonging to a specified interval. The co-existence that is brought can either be stable or saddle depending on the ecosystem parameters. 
Increasing the quantity of the additional food beyond a critical level, with quality belongs to a specified interval, all the solutions of the system will be driven to a state without the economically damaging species.

Finally, we conclude that the modified version of additional food provided Lokta-Volterra competition model exhibits very interesting dynamics. By manipulating quality and quantity of additional food that is supplied to noneconomically damaging species, it is possible to control the dynamics of the system. It is possible to drive the levels of both the pest to a desired level within specified limits. By providing additional food having a specified characteristics, we can, eliminate economically damaging pest by enhancing the competition by supplying right kind of additional food to the non-economically damaging species. Finally, it can be concluded that the current study finds its vital applications in the biological pest control programs using competitive species for controlling the pest.

\section{References}

[1] Abrams, P. A., Defining and measuring the impact of dynamic traits on interspecific interactions, Ecology, 88, No. 10 (2007), 2555-2562.

[2] Abrams, P.A. and M. Nakajima, Does competition between resources reverse the competition between their consumers? Variations on two themes by Vandermeer, Am. Nat., 170, No. 5 (2007), 744-757.

[3] Abrams, P.A. and Cortez, M.H., The many potential indirect interactions between predators that share competing prey, Ecology, 85, No. 4 (2015), 625-641.

[4] Ayala, F.J. and Gilpin M.E., Competition between species: Theoretical Models and Experimental Tests, Theor. Popul. Biol., 4, No. 3 (1973), 331-356.

[5] Bader, A.E., K.M. Heinz and R.A. Wharton, and C.E. Borgan, Assessment of interspecific interactions among parasitoids on the outcome of inoculative biological control of leafminers attaching chrysanthemum, Biol. Control, 39, No. 3 (2006), 441-452.

[6] Borgan, C.E., K.M. Heinz and M.A. Ciomperlik, Interspecific competition among insect parasitoids: field experiment with whiteflies as hosts in cotton, Ecology, 83 No. 3 (2002), 653-668.

[7] Cheng, E.Y., Problems of control of insecticide-resistant plutella xylostella, Pestic. Sci., 23, No. 2 (1988), 177-178.

[8] Connell J.H., On the prevalence and relative importance of interspecific competition: Evidence from field experiments, Am. Nat., 122, No. 5 (1983), 661-696.

[9] Courchamp, F., C. Suppo, E. Fromont and C. Bouloux, Dynamics of two feline retroviruses (FIV and FeLv) within one population of cats. Proc. of the Royal Society of London B: Biological Sciences, 264, (1997), 785-794.

[10] Courchamp, F. and G. Sugihara, Modelling the biological control of an alien predtor to protect island species from extinction, Ecol. Appl., 9, No. 1 (1999), 112-123. 
[11] Coll, M., Parasitoids in diversified intercropped systems. Inc: C.H. Pickett and R.Bugg (ed.), Enhancing biological control: Habitat management to promote natural enemies of argicultural pets, University of California Press, 85-120, Berkely, USA (1998).

[12] Coll, M., Feeding on non-prey resources by natural enemies. In: J.G. Lundgren, Relationships of natural enemies and non-prey foods, Springer, pp. ix-xxiii, USA (2009).

[13] Gause, G.F., The struggle for existence, The Williams \& Wilkins Company, Baltimore, USA (1934).

[14] Gill, H.K. and H. Garg, Pesticides: Environment impacts and management strategies, In: M.L. Larramendy and S. Soloneski(ed.), Pesticides toxic Aspects, Intech, Croapia (2014).

[15] Hagen, K.S., Ecosystem analysis: plant cultivars (HPR), entromophagous species and food supplements. In: D.J. Boethel and R.D. Eikenbary (ed.), Interactions of plant resistance and parasitoids and predators of insects, Halsted, New York (1986).

[16] Harmon, J.P., Indirect interactions among a generalist predator and its multiple foods, Ph.D thesis, St.Paul, Univ. of Minnesota, USA (2003).

[17] Harwood, J.D. and J.J. Obrycki, The role of alternative prey in sustaining predator populations, proc. of second international symposium on biological control of arthropods, 2, (2005), 453-462.

[18] Holt, R.D., Predation, apparent competition, and the structure of prey communities, Theor. Popul. Biol., 12, No.2 (1977), 197-229.

[19] Hougen-Eitzman, D. and R. Karban, Mechanisms of interspecific competition that result in successful control of pacific mites following inoculations of willamette mites on grapevines, Oecologia, 103, No. 2 (1995), 157-161.

[20] Kot, M., Elements of Mathematical Ecology, Cambridge University Press, Cambridge, UK (2003).

[21] Levine, S.H., Competitive interaction in ecosystems, Am. Nat., 110, No. 976 (1976), 903-910.

[22] Levins, R., Evolution in changing environments, Princeton University Press, New Jersey, USA (1968).

[23] Lundgren, J.G., Relationships of Natural Enemies and Non-prey Foods, Springer-Verlag, Netherlands (2009).

[24] MacArthur, R., Species packing and competitive equilibrium for many species, Theor. Popul. Biol., 1, No. 1 (1970), 1-11.

[25] Mustu, M. and N. Kilincer, Interspecific competition between Anagyrus pseudococci and Leptomastix dactylopii, parasitoids of the vine mealybug Planococcus ficus, Biocontrol, 60, No. 4 (2015) 485-493.

[26] Murdoch, W.W., J. Chesson and P.L. Chesson, Biological control in theory and practice, Am. Nat., 125, No. 3 (1985), 344-366.

[27] Orr, D. and S. Lahiri, Biological control of Insect pest in crops. In: D.P. Abrol (ed.), Intergrated Pest Management: Current Concepts and Ecological Perspectives, Elsevier, San Diego (2014), 531-548.

[28] Prasad, B.S.R.V., Malay Banerjee and P.D.N. Srinivaus, Dynamics of additional food provided predator-prey system with mutually interfering predators, Math. Biosci., 246, No. 1(2013), 176-190. 
[29] Srinivasu, P.D.N., B.S.R.V. Prasad and M. Venkatesulu, Biological control through provision of additional food to predators: a theoretical study, Theor. Popul. Biol., 72, No. 1(2007), 111-120.

[30] Srinivasu, P.D.N. and B.S.R.V. Prasad, Time optimal control of an additional food provided predator-prey system with application to pest management and bioloigcal conservation, J. Math. Biol., 60, No. 4(2010), 591-613.

[31] Srinivasu, P.D.N. and B.S.R.V. Prasad, Role of quantity of additional food to predators as a control in predator-prey systems with relevance to pest management and biological conservation, Bull. Math. Biol, 73, No. 10(2012) 2249-2276.

[32] Tilman, D., The importance of the mechanisms of interspecific competition, Am. Nat., 129, No. 5(1987), 769-774.

[33] van Emden, H.F., The role of uncultivated land in the biology of crop pests and beneficial insects, Sci. Hortic., 17, (1965), 121-136.

[34] Wäckers, F.L. and P.C.J. van Rijn, Food for protection: an introduction. In: F.L. Wäckers, P.C.J. van Rijn and J. Bruin (ed.), Plant-Provided Food for Carnivorous Insects: A Protective Mutualism and Its Applications, Cambridge University Press, Cambridge(2005).

[35] Zhou, Z.S., Z.P. Chen and Z.F. Xu, Niches and interspecific competitive relationships of the parasitoids, Microplitis prodeniae and Camploetis chlorideae, of the Oriental leafworm moth, Spodoptera litura, in tobacco, J. Insect Science, 10, No. 10(2010), 1-12. 
